\title{
LIMITES DE PRECAUÇÃO E DE CONTROLE EM ANÁLISES NUTRICIONAIS DE ESPÉCIES FLORESTAIS ${ }^{1}$
}

Alessandro Dal Col Lúcio², Rafael Augusto Rossato de Rossato³, Mauro Valmir Schumacher², Fabiano de Oliveira Fortes ${ }^{2}$, Lindolfo Storck ${ }^{2}$ e Rudi Witschoreck ${ }^{2}$

\begin{abstract}
RESUMO - Este trabalho teve por objetivo estimar os limites de precaução e de controle para os macros e micronutrientes presentes em diferentes componentes da biomassa de Acacia mearnsii, Eucalyptus spp. e Pinus taeda. As 40.183 análises foram separadas por elemento químico, componente e espécie. Foram estabelecidos os limites com base nos resultados dos testes de aderência à distribuição normal e nos critérios de classificação. Os resultados dos teores médios obtidos ficaram dentro da faixa de valores encontrados na literatura para as espécies florestais estudadas, componentes da biomassa e elementos químicos. Os teores dos elementos químicos foram diferentes entre as espécies para cada tipo de componente analisado. Independentemente da espécie florestal estudada, as folhas e acículas apresentam maiores teores, enquanto o lenho, os menores. Os limites estimados, propostos neste trabalho, permitem maior controle na classificação dos resultados, aumentando a confiabilidade nas análises nutricionais.
\end{abstract}

Palavras-chave: Acacia mearnsii, Eucalyptus spp., Pinus taeda e controle de qualidade.

\section{LIMITS OF CAUTION AND CONTROL IN NUTRITIONAL ANALYSIS OF FOREST SPECIES}

\begin{abstract}
The paper had as its objective to estimate the precaution limits and control for macro and micro nutrients, present in different components of the biomass, of Acacia mearnsii, Eucalyptus spp. and Pinus taeda. The 40,183 analyses were separated by chemical element, component and species. The limits with base in the results of the tests of adherence to the normal distribution and in the classification criteria were established. The results of the obtained medium tenors are inside of the strip of values found in the literature for the studied forest species, components of the biomass and chemical elements. The levels of the chemical elements are different among the species for each type of analyzed component. Independent of the studied forest species, the leaves and needles presented larger values while the buds trunk smaller. The limits, proposed in the work, allow greater control in the classification of the results, increasing the reliability in the nutritional analyses.
\end{abstract}

Keywords: Acacia mearnsii, Eucalyptus spp., Pinus taeda and quality control.

\section{INTRODUÇÃO}

As análises de nutrientes em tecidos vegetais de espécies florestais estão sujeitas aos diversos tipos de erros e, como são inevitáveis, o importante é mantê-los sob controle (CANTARELLA et al., 2001), pois os valores ou medidas resultantes das análises devem ser exatos e precisos, indicando que o valor é o mais próximo possível do verdadeiro e pode ser reproduzido em análises independentes. Esses erros ocorrem durante o processo de preparação das amostras e nas determinações quantitativas ou qualitativas. Ao obter resultados analíticos de amostras químicas, os valores podem apresentar erros aleatórios, que só serão detectados

\footnotetext{
${ }^{1}$ Recebido em 03.07.2007 e aceito para publicação em 02.03.2010.

${ }^{2}$ Universidade Federal de Santa Maria, UFSM, Brasil. E-mail:<adlucio@smail.ufsm.br>, <schuma@pq.cnpq.br>, <fabfortes@yahoo.com.br>,<lindolfo@smail.ufsm.br>e <rwitschoreck@yahoo.com.br>.

${ }^{3}$ Aracruz Celulose S.A., Brasil. E-mail: <rarossato@aracruz.com.br>.
} 
por meio de procedimentos estatísticos, ou sistemáticos, causados por equipamentos, calibrações ou procedimentos. Os erros podem afetar a análise de dados, prejudicando as estimativas dos efeitos de tratamentos, além de mascarar relações entre variáveis, conforme descreveram Lorentz et al. (2006).

A determinação de valores de controle é a melhor alternativa para a indicação imediata de desvios da qualidade dentro de limites pré determinados, evitando a baixa precisão ou exatidão de um resultado. O controle de resultados de laboratório, via definição dos limites de controle de resultados, visa decidir se os registros produzidos são aceitáveis ou devem ser descartados. Os valores encontrados devem girar em torno da média, não ultrapassando os limites estabelecidos no intervalo de confiança (FEIGENBAUM, 1994). Variações como ajuste de instrumentos, erros nas medidas de massa, volume e tempo, contaminação dos reagentes, variações nos números de fótons emitidos ou absorvidos, entre outros, contribuem para a incerteza de qualquer medida ou determinação. Por isso, ao ser adotado o controle estatístico da qualidade para estabelecer e manter os níveis de qualidade, tanto operacionais quanto experimentais, é possível estabelecer critérios para a melhoria da qualidade dos serviços prestados e da elaboração do produto final (ARACRUZ CELULOSE, 2004; JACOVINE et al., 2005).

Com a dificuldade no controle de certos processos contínuos, podem ser utilizados gráficos ou tabelas de controle, que têm como função principal a indicação imediata de tendências de desvios da qualidade nos limites pré determinados (ELEOTERIO et al., 1996). Além de indicarem a ultrapassagem desses limites, servem, principalmente, para o controle de influências responsáveis pela qualidade insatisfatória dos resultados, indicando de forma simples, visual, prática e eficiente, o eventual descontrole do processo (BRAVO, 1995).

A distribuição dos nutrientes nas diversas partes e componentes de uma árvore é variável, pois um tecido pode conter teores variados de um nutriente em função da sua distribuição na planta. O conhecimento dessa distribuição pode contribuir para o estudo das espécies, a fim de suprir suas necessidades nutricionais, favorecendo um ciclo nutricional fechado e reduzindo a exportação de nutrientes, contribuindo, dessa forma, para a sustentabilidade do ecossistema florestal. Diante disso, autores como Dell et al. (1995), Caldeira et al. (2000), Neves (2000), Schumacher et al. (2002), Barrichelo
(2003), Gonçalves et al. (2004), Bizon (2005) e Witschoreck (2008). salientaram o status nutricional das espécies em diversas condições, o que possibilita melhor compreensão das faixas de nutrientes classificadas nos limites de controle, bem como orientar estudos futuros envolvendo classificação de sítios e determinação de nutrientes em espécies florestais, refletindo o estado nutricional da planta e a fertilidade do solo, sendo ferramenta importante para avaliar e determinar as condições de crescimento de povoamento florestal. A quantificação desses teores pode influenciar o manejo adequado que resulte em grande economia de nutrientes para o estoque da área, com elevada repercussão sobre o seu potencial produtivo (GONÇALVES, 1995).

Em árvores com diferentes idades foram obtidas diferenças significativas para N, K, Fe e Mn, enquanto o P, Ca e Mg não variaram significativamente(REISSMANN et al., 1976). De Hoogh (1981) não encontrou diferenças entre árvores mais novas e mais velhas, enquanto Simões et al. (1973) obtiveram valores baixos para N e K nas árvores mais novas, em comparação com as mais velhas. A distribuição vertical de nutrientes na copa das árvores está relacionada com os diversos tipos de solos e condições fisiológicas locais, em que elementos como o P, K, Ca e Mg mostram variações regulares de acordo com a posição das amostras de folhas na copa (De HOOGH, 1981). Esse autor também destacou condições anormais de umidade que influenciam na redução dos teores de N, P, Zn e Cu após seca extrema. Variações sazonais significativas nos teores de nutrientes em uma mesma árvore foram observadas por Reissmann et al. (1976), em que os níveis de N, P e K mostraram oscilação nas amostras coletadas entre o verão e o inverno. Binkley (1986) também demonstrou pequenas flutuações nos teores de $\mathrm{N}$ em acículas novas e velhas em relação à época do ano.

O trabalho teve como objetivo estimar os limites de precaução e de controle dos teores de macro e micronutrientes, presentes em diferentes componentes da biomassa, em três espécies florestais.

\section{MATERIAL E MÉTODOS}

Utilizaram-se os resultados das análises realizadas entre os anos de 2003 e 2007 no Laboratório de Ecologia Florestal do Departamento de Ciências Florestais, do Centro de Ciências Rurais da Universidade Federal de Santa Maria, das espécies Acacia mearnssi De Wild., Eucalyptus spp. e Pinus taeda L. Os dados são referentes as análises 
nutricionais de 3.841 amostras, com 40.183 determinações, dos seguintes elementos químicos: N, P, K, Ca, Mg e $\mathrm{S}\left(\mathrm{em} \mathrm{g} \mathrm{kg}^{-1}\right.$ ), B, Cu, Fe, Mn e Zn (em mg kg${ }^{-1}$ ). Os resultados analíticos são de amostras de tecido vegetal coletadas segundo diferentes metodologias e oriundas dos seguintes componentes da biomassa: folha (acícula no caso de Pinus taeda), proveniente de coletas com o objetivo de avaliar o estado nutricional (folhas recém-maduras), bem como amostras aleatórias de estudos ecológicos, com o objetivo de quantificar o estoque de nutrientes; galho com casca, vivo e morto, de diferentes diâmetros e posições na copa das árvores; e casca e lenho, coletados em diferentes alturas ao longo do tronco.

Quanto aos procedimentos analíticos, o $\mathrm{N}$ foi determinado pelo método Kjeldhal, no extrato de digestão sulfúrica; B por espectrofotometria com digestão seca; e os demais elementos, no extrato de digestão nítricoperclórica, sendo $\mathrm{Ca}, \mathrm{Mg}, \mathrm{Cu}, \mathrm{Fe}, \mathrm{Mn}$ e $\mathrm{Zn}$ por espectrofotometria de absorção atômica, $\mathrm{P}$ por espectrofotometria, K por fotometria de chama e S por turbidimetria (MALAVOLTA et al., 1997).

Foram estimados a média e o desvio-padrão de cada nutriente, em cada componente e espécie estudados, bem como o número de observações (n). Os dados foram submetidos ao teste de Lilliefors (CAMPOS, 1983), autor que estudou o ajuste dos dados à distribuição normal. Nos casos em que os valores se aderiram à distribuição normal, adotou-se o critério para determinação de limites estabelecido por Lúcio et al. (1999), e nos casos da não aderência à distribuição normal, foram definidas as classes com base na média e desvio-padrão, de acordo com o critério descrito em Feigenbaum (1994) (Tabela 1). Com os valores centrais e dos limites inferiores e superiores estabelecidos, confeccionaram-se as tabelas com os limites de controle e de precaução para cada nutriente, componente vegetal e espécie.
Em todas as análises estatísticas foi utilizado o software estatístico SAEG 9.1 (SAEG, 2007), adotando se $5 \%$ de probabilidade de erro.

\section{RESULTADOS E DISCUSSÃO}

A aderência à distribuição normal foi de 32,88\%, quando consideradas todas as espécies, componentes vegetais e elemento químico. Quando dentro de cada espécie, foram obtidos 0, 52,00 e 41,88\% de aderência à distribuição normal, respectivamente para Eucalyptus spp., Acacia mearnsii e Pinus taeda (Tabelas 2, 3 e 4).

A quantidade de nutrientes nas plantas variou bastante em função da espécie e genótipo, idade da planta, sítio, época do ano e da parte da planta analisada, concordando com resultados de Pallardy (2008). Segundo Bellote e Silva (2004), a concentração dos nutrientes nos componentes da biomassa está relacionada com suas funções, apresentando, geralmente, o seguinte gradiente: folha casca ramo tronco (alburno cerne).

A folha (acícula) é o grande centro metabólico da planta, o que fica evidente pelo predomínio melas dos teores mais elevados de nutrientes (MARSCHNER, 1995) independentemente da espécie florestal. Entretanto, os teores mais baixos estão associados a componentes que têm função mais estrutural ou de condução, como é o caso do lenho e dos galhos (Tabelas 2, 3 e 4).

No caso dos macronutrientes, somente Ca na Acacia mearnsii e Ca e Mg no Eucalyptus spp. apresentaram teores mais elevados na casca que na folha, enquanto no Pinus taeda todos os nutrientes estavam mais concentrados na acícula (Tabelas 2, 3 e 4). Do mesmo modo, em se tratando de micronutrientes, à exceção de Mn na casca de Eucalyptus spp. e de Cu e Fe respectivamente, no galho vivo e casca de Pinus taeda, os teores são mais elevados nas folhas. Por seu turno,

Tabela 1 -Critérios de limites de classe para classificação dos resultados nas análises nutricionais. Table 1 - Criteria of class limits for classification of the results in nutritional analysis.

\begin{tabular}{|c|c|c|}
\hline Limites de classe (LÚCIO, et al. 1999) & Limites de classe (FEIGENBAUM, 1994) & Classificação dos valores \\
\hline Menor que $\mathrm{X}_{1}=-1,67 \mathrm{~s}_{\mathrm{v}}+\bar{x}_{\mathrm{v}}^{*}$ & $\mathrm{LCS}_{\mathrm{x}}=\bar{x}_{\mathrm{v}}-\mathrm{A}_{3} 3 \mathrm{~s}_{\mathrm{v}}{ }^{*}$ & Muito baixo \\
\hline Entre $\mathrm{X}_{1}$ e $\mathrm{X}_{2}=-0,64 \mathrm{~s}_{\mathrm{v}}+\vec{X}_{\mathrm{v}}$ & $\operatorname{LPS}_{\mathrm{x}}=\vec{x}_{\mathrm{v}}-\mathrm{A}_{3} 2 \mathrm{~s}_{\mathrm{v}}$ & Baixo \\
\hline Entre $\mathrm{X}_{2}$ e $\mathrm{X}_{3}=0,64 \mathrm{~s}_{\mathrm{V}}+\bar{x}_{\mathrm{V}}$ & Entre $\mathrm{LPS}_{\mathrm{x}}$ e $\mathrm{LPI}_{\mathrm{x}}$ & Médio \\
\hline Entre $\mathrm{X}_{3}$ e $\mathrm{X}_{4}=1,67 \mathrm{~s}_{\mathrm{v}}+\overline{\boldsymbol{X}}_{\mathrm{v}}$ & $\mathrm{LPI}_{\mathrm{x}}=\bar{x}_{\mathrm{v}}+\mathrm{A}_{3} 2 \mathrm{~s}_{\mathrm{v}}$ & Alto \\
\hline Maior que $\mathrm{X}_{4}^{4}=1,67 \mathrm{~s}_{\mathrm{v}}+\bar{X}_{\mathrm{v}}^{\mathrm{v}}$ & $\mathrm{LCI}_{\mathrm{X}}=\overline{\boldsymbol{X}}_{\mathrm{v}}^{\mathrm{v}}+\mathrm{A}_{3} 3 \mathrm{~s}_{\mathrm{v}}$ & Muito alto \\
\hline
\end{tabular}

$* \bar{x}=$ média e s = desvio- padrão dos resultados (V); LCS = limite de controle superior; LPS= limite de precaução superior; VC= valor central; LCI = limite de controle inferior; LPI = limite de precaução inferior; e A3 = tabelado em função do número de observações (n).

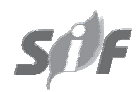

Revista Árvore, Viçosa-MG, v.34, n.3, p.529-537, 2010 
o lenho é o componente com os menores teores, à exceção apenas de K, S e Mn no Pinus taeda, Fe no Eucalyptus spp. e Cu na Acacia mearnssi (Tabelas 2, 3 e 4).

A variação na concentração de nutrientes, entre componentes e dentro de um mesmo componente, tende a se intensificar com a idade, em decorrência da ciclagem interna (ciclagem bioquímica), que desloca nutrientes de tecidos senescentes para regiões com maior atividade metabólica (REIS e BARROS, 1990; PALLARDY, 2008). Esse processo é mais intenso no tronco, onde ocorre a transformação de alburno em cerne. Segundo Burger e Richter (1991), o alburno, localizado nas camadas mais periféricas do tronco, constitui-se em tecido mais ativo, fisiologicamente, que o cerne, responsável pelo transporte ascendente de líquidos na árvore e constituído por células

Tabela 2 - Número de observações (n), média ( $\bar{x}$ ) e desvio- padrão (DP) dos teores de nutrientes obtidos em tecidos vegetais de Acacia mearnsii. Santa Maria ,- RS, 2007.

Table 2 - Number of observations (n), means ( $\bar{x})$ and standard deviation (DP) of the levels of nutrients obtained in vegetable tissues of Acacia mearnsii. Santa Maria, - RS, 2007.

\begin{tabular}{|c|c|c|c|c|c|c|c|c|c|c|c|c|}
\hline \multirow[t]{2}{*}{ Tecido vegetal } & & $\mathrm{N}$ & $\mathrm{P}$ & K & $\mathrm{Ca}$ & $\mathrm{Mg}$ & $\mathrm{S}$ & B & $\mathrm{Cu}$ & $\mathrm{Fe}$ & $\mathrm{Mn}$ & $\mathrm{Zn}$ \\
\hline & & \multicolumn{6}{|c|}{$\mathrm{g} \mathrm{kg}^{-1}$} & \multicolumn{5}{|c|}{$\mathrm{mg} \mathrm{kg}^{-1}$} \\
\hline \multirow[t]{3}{*}{ Folha } & $\mathrm{n}$ & 111 & $109 *$ & 111 & 109 & 109 & $110^{*}$ & 100 & $99 *$ & 90 & 101 & $93^{*}$ \\
\hline & $x$ & 32,05 & 1,66 & 9,59 & 7,06 & 2,19 & 1,38 & 32,39 & 7,73 & 166,37 & 270,65 & 19,47 \\
\hline & $\mathrm{DP}$ & 6,95 & 0,46 & 3,33 & 1,93 & 0,49 & 0,38 & 12,03 & 2,73 & 98,33 & 311,75 & 5,03 \\
\hline \multirow[t]{3}{*}{ Lenho } & $\underline{\mathrm{n}}$ & 155 & 151 & 120 & 157 & $157 *$ & 157 & 53 & 49 & 48 & 53 & 53 \\
\hline & $x$ & 2,21 & 0,17 & 1,81 & 2,42 & 0,44 & 0,22 & 6,34 & 2,61 & 18,72 & 15,44 & 8,38 \\
\hline & DP & 1,87 & 0,20 & 1,39 & 2,04 & 0,19 & 0,10 & 2,04 & 1,91 & 17,93 & 15,48 & 4,54 \\
\hline \multirow[t]{3}{*}{$\overline{\text { Cascan }}$} & $\underline{\mathrm{n}}$ & $137 *$ & $137^{*}$ & $104 *$ & $138^{*}$ & $136^{*}$ & $138^{*}$ & $41^{*}$ & 37 & 40 & 41 & $41^{*}$ \\
\hline & $x$ & 10,46 & 0,40 & 5,89 & 8,70 & 1,60 & 0,28 & 16,57 & 1,96 & 52,76 & 35,40 & 14,68 \\
\hline & DP & 1,57 & 0,11 & 1,21 & 3,01 & 0,44 & 0,10 & 3,41 & 0,84 & 28,29 & 26,08 & 4,36 \\
\hline \multirow[t]{3}{*}{ Galho morto } & $\underline{\mathrm{n}}$ & $10^{*}$ & $10 *$ & $10^{*}$ & $10 *$ & $10 *$ & $10^{*}$ & - & - & - & - & - \\
\hline & $\bar{x}$ & 8,73 & 0,28 & 2,67 & 5,70 & 1,99 & 0,42 & - & - & - & - & 一- \\
\hline & DP & 1,84 & 0,10 & 1,17 & 1,30 & 0,39 & 0,16 & -- & -- & -- & -- & - \\
\hline \multirow[t]{3}{*}{ Galho vivo } & $\underline{\mathrm{n}}$ & $45^{*}$ & $44^{*}$ & $26 *$ & $45^{*}$ & 45 & $45^{*}$ & $36^{*}$ & 30 & $36^{*}$ & 31 & 36 \\
\hline & $\bar{x}$ & 8,81 & 0,78 & 6,62 & 4,50 & 1,16 & 0,43 & 13,00 & 3,80 & 43,51 & 43,26 & 18,57 \\
\hline & DP & 2,99 & 0,33 & 2,28 & 1,20 & 0,40 & 0,19 & 2,04 & 2,32 & 21,91 & 33,41 & 8,55 \\
\hline
\end{tabular}

*: Distribuição normal pelo teste de Lilliefors a 5 \% de probabilidade de erro.

Tabela 3 - Número de observações (n), média $(\bar{x})$ e desvio- padrão (DP) dos teores de nutrientes obtidos em tecidos vegetais de Eucalyptus spp. Santa Maria, - RS, 2007.

Table 3 - Number of observations (n), means $(\bar{X})$ and standard deviation (DP) of the levels of nutrients obtained in vegetable tissues of Eucalyptus spp. Santa Maria, - RS, 2007.

\begin{tabular}{|c|c|c|c|c|c|c|c|c|c|c|c|c|}
\hline \multicolumn{2}{|c|}{ Tecido vegetal } & $\mathrm{N}$ & $\mathrm{P}$ & $\mathrm{K}$ & $\mathrm{Ca}$ & $\mathrm{Mg}$ & $\mathrm{S}$ & $\mathrm{B}$ & $\mathrm{Cu}$ & $\mathrm{Fe}$ & $\mathrm{Mn}$ & $\mathrm{Zn}$ \\
\hline & & \multicolumn{6}{|c|}{$\mathrm{g} \mathrm{kg}^{-1}$} & \multicolumn{5}{|c|}{$\mathrm{mg} \mathrm{kg}^{-1}$} \\
\hline \multirow[t]{3}{*}{ Folha } & $\underline{\mathrm{n}}$ & 1.466 & 1.470 & 1.469 & 1.469 & 1.469 & 1.468 & 1.473 & 1.469 & 1.468 & 1.474 & 1.465 \\
\hline & $\bar{x}$ & 24,16 & 1,45 & 10,08 & 8,00 & 2,43 & 1,29 & 33,86 & 7,13 & 71,43 & 918,00 & 19,40 \\
\hline & $\mathrm{DP}$ & 5,45 & 0,43 & 3,49 & 2,80 & 0,67 & 0,27 & 14,71 & 2,89 & 37,83 & 638,58 & 7,55 \\
\hline \multirow[t]{3}{*}{ Lenho } & $\underline{\mathrm{n}}$ & 481 & 480 & 481 & 478 & 475 & 472 & 481 & 479 & 454 & 478 & 481 \\
\hline & $\bar{x}$ & 2,09 & 0,25 & 2,60 & 1,43 & 0,49 & 0,23 & 4,71 & 2,80 & 40,99 & 93,17 & 5,25 \\
\hline & $\mathrm{DP}$ & 0,92 & 0,25 & 1,22 & 0,88 & 0,20 & 0,09 & 2,90 & 1,61 & 24,75 & 103,07 & 5,21 \\
\hline \multirow[t]{3}{*}{ Casca } & $\underline{n}$ & 468 & 468 & 467 & 468 & 468 & 465 & 468 & 462 & 450 & 464 & 469 \\
\hline & $\bar{x}$ & 4,57 & 0,53 & 5,77 & 22,21 & 3,30 & 0,37 & 20,70 & 3,40 & 43,57 & 1006,59 & 9,18 \\
\hline & $\mathrm{DP}$ & 0,88 & 0,13 & 1,88 & 7,70 & 1,18 & 0,13 & 6,22 & 1,27 & 24,31 & 489,03 & 5,13 \\
\hline \multirow[t]{3}{*}{ Galho vivo } & $\underline{\mathrm{n}}$ & 479 & 477 & 471 & 476 & 478 & 478 & 479 & 478 & 449 & 474 & 479 \\
\hline & $\bar{x}$ & 4,37 & 0,57 & 4,97 & 10,80 & 1,68 & 0,35 & 12,13 & 5,76 & 37,43 & 574,55 & 11,71 \\
\hline & $\mathrm{DP}$ & 1,34 & 0,31 & 1,63 & 4,73 & 0,51 & 0,13 & 2,83 & 1,88 & 21,40 & 315,43 & 5,85 \\
\hline
\end{tabular}

*: Distribuição normal pelo teste de Lilliefors a 5\% de probabilidade de erro.

Revista Árvore, Viçosa-MG, v.34, n.3, p.529-537, 2010 
Tabela 4-Número de observações (n), média $(\bar{X})$ e desvio- padrão (DP) dos teores de nutrientes obtidos em tecidos vegetais de Pinus taeda. Santa Maria, - RS, 2007.

Table 4 - Number of observations (n), means $(\bar{x})$ and standard deviation (DP) of the levels of nutrients obtained in vegetable tissues of Pinus taeda. Santa Maria, - RS, 2007.

\begin{tabular}{|c|c|c|c|c|c|c|c|c|c|c|c|c|}
\hline \multirow[t]{2}{*}{ Tecido vegetal } & & $\mathrm{N}$ & $\mathrm{P}$ & $\mathrm{K}$ & $\mathrm{Ca}$ & $\mathrm{Mg}$ & $\mathrm{S}$ & $\mathrm{B}$ & $\mathrm{Cu}$ & $\mathrm{Fe}$ & $\mathrm{Mn}$ & $\mathrm{Zn}$ \\
\hline & & \multicolumn{6}{|c|}{$\mathrm{g} \mathrm{kg}^{-1}$} & \multicolumn{5}{|c|}{$\mathrm{mg} \mathrm{kg}^{-1}$} \\
\hline \multirow[t]{3}{*}{ Acícula } & $\underline{\mathrm{n}}$ & 41 & $39 *$ & 39 & $43^{*}$ & 40 & 39 & $40^{*}$ & $41^{*}$ & $39^{*}$ & $40^{*}$ & $33^{*}$ \\
\hline & $\bar{x}$ & 11,39 & 1,05 & 4,70 & 3,13 & 1,23 & 0,92 & 18,27 & 4,47 & 167,27 & 267,95 & 23,26 \\
\hline & $\mathrm{DP}$ & 3,05 & 0,24 & 2,26 & 0,87 & 0,29 & 0,41 & 7,29 & 1,08 & 66,36 & 160,02 & 4,71 \\
\hline \multirow[t]{3}{*}{$\overline{\text { Lenho }}$} & $n$ & 163 & 160 & $161 *$ & 163 & 163 & 155 & 143 & 159 & 128 & 154 & 141 \\
\hline & $\bar{x}$ & 1,43 & 0,11 & 0,60 & 0,70 & 0,28 & 0,38 & 5,53 & 1,96 & 25,28 & 59,37 & 6,23 \\
\hline & DP & 0,47 & 0,06 & 0,20 & 0,16 & 0,08 & 0,10 & 2,25 & 0,89 & 18,09 & 39,52 & 3,64 \\
\hline \multirow[t]{3}{*}{$\overline{\text { Casca }}$} & $\underline{n}$ & 157 & 158 & 149 & 146 & 157 & 156 & 157 & $133^{*}$ & $145^{*}$ & 153 & $\overline{141}$ \\
\hline & $\bar{x}$ & 3,45 & 0,35 & 1,41 & 1,48 & 0,59 & 0,44 & 12,17 & 4,43 & 170,13 & 46,00 & 22,05 \\
\hline & $\mathrm{DP}$ & 1,41 & 0,21 & 0,98 & 1,02 & 0,32 & 0,14 & 4,54 & 1,06 & 87,92 & 21,19 & 13,65 \\
\hline \multirow[t]{3}{*}{ Galho vivo } & $\underline{n}$ & $40 *$ & 42 & 38 & 41 & $41 *$ & 42 & $41 *$ & $41 *$ & $42 *$ & $42 *$ & $40 *$ \\
\hline & $\bar{x}$ & 3,28 & 0,38 & 1,42 & 1,76 & 0,58 & 0,45 & 9,59 & 5,06 & 74,81 & 136,51 & 17,36 \\
\hline & $\mathrm{DP}$ & 1,41 & 0,22 & 1,10 & 0,81 & 0,16 & 0,16 & 2,33 & 2,11 & 35,49 & 75,42 & 5,92 \\
\hline \multirow[t]{3}{*}{ Galho morto } & $n$ & 38 & 36 & $35 *$ & 38 & $36 *$ & $38 *$ & $37 *$ & $37 *$ & $36^{*}$ & 37 & 36 \\
\hline & $\bar{x}$ & 1,94 & 0,13 & 0,24 & 2,33 & 0,40 & 0,35 & 9,72 & 4,35 & 120,67 & 164,26 & 11,78 \\
\hline & DP & 0,75 & 0,08 & 0,12 & 0,99 & 0,10 & 0,14 & 2,04 & 1,94 & 60,36 & 119,77 & 7,99 \\
\hline
\end{tabular}

*: Distribuição normal pelo teste de Lilliefors a 5\% de probabilidade de erro.

parenquimáticas vivas que encerram substâncias nutritivas como amido, açúcares, proteínas etc. Como a proporção de alburno aumenta da base da árvore em direção ao ápice, é possível verificar elevação na concentração, para a maioria dos nutrientes, na madeira do tronco, na região terminal do tronco.

A diferença de concentração de nutrientes em amostras de casca e madeira coletadas em diferentes posições ao longo do tronco da árvore depende, principalmente, da mobilidade dos nutrientes no tecido vegetal. Assim, enquanto os elementos imóveis ou pouco móveis se acumulam na base ou não variam expressivamente ao longo do tronco, os nutrientes com alta mobilidade apresentam os maiores teores na extremidade superior do tronco (ZEN et al., 1981; ANDRAE e KRAPFENBAUER, 1983; WITSCHORECK, 2008).

De forma análoga, a transformação de alburno em cerne também imprime variação no sentido transversal do tronco, como verificado por Silva (1996) em Eucalyptus grandis aos 7 anos de idade, principalmente quanto ao conteúdo de $\mathrm{N}, \mathrm{P}$ e $\mathrm{K}$.

A diferença na concentração dos nutrientes entre galho vivo e galho morto é outra evidência da ciclagem interna e da diferença de mobilidade dos nutrientes no tecido vegetal. Para exemplificar, enquanto o processo de senescência provoca redução nos teores de nutrientes como P e K (altamente móveis no tecido vegetal), o Ca (imóvel) apresentou comportamento distinto, tendo teores mais altos no galho morto.

Cabe ressaltar a importância de se verificar a compatibilidade dos métodos analíticos utilização de limites de precaução e de controle. Nesse sentido, em função de diferenças analíticas e do grande número de fatores que interferem na concentração de nutrientes nos vários componentes da biomassa florestal, é preciso bastante cautela na utilização de valores produzidos em outras condições, principalmente fora do país.

Os resultados médios mostrados nas Tabelas 2, 3 e 4 são condizentes com a faixa de valores encontrados na literatura para todas as espécies florestais, tecidos e nutrientes. Dessa forma, o tipo de resposta obtida a partir de análise de tecido de determinada espécie florestal será dependente da espécie analisada, bem como da parte da planta, confirmação óbvia, mas que sustenta a classificação dos limites dos elementos químicos com base nos resultados das análises. Os valores diferenciados, quando comparados com as médias dos resultados de literatura, são explicados pelos fatores expostos pelos autores, o que comprova a necessidade de realizar as análises das distribuições específicas para cada caso e a separação dos resultados agrupados nos componentes vegetais de cada espécie.

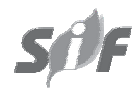

Revista Árvore, Viçosa-MG, v.34, n.3, p.529-537, 2010 
As Tabelas 5, 6 e 7 apresentam os limites de precaução e controle, inferiores e superiores dos teores de cada elemento químico e espécie florestal, fornecendo condições mais adequadas aos usuários de análises laboratoriais em avaliar os resultados e tomar decisão mais confiável e consistente em relação aos valores obtidos. Com essa avaliação há, ainda, a possibilidade em verificar se os valores observados acima ou abaixo

Tabela 5 - Valores de classe obtidos dos limites de controle (LC) e de precaução (LP) superiores (S) e inferiores (I) para resultados dos teores de nutrientes obtidos em tecidos vegetais de Acacia mearnsii. Santa Maria, RS, 2007.

Table 5 - Class values obtained of the limits of control (LC), caution (LP) superiors (S) and lower (I) to the levels of nutrients results obtained in vegetables tissues of Acacia mearnsii. Santa Maria, RS, 2007.

\begin{tabular}{|c|c|c|c|c|c|c|c|c|c|c|c|c|}
\hline \multirow[t]{2}{*}{ Tecido vegetal } & \multirow[b]{2}{*}{ Limites } & $\mathrm{N}$ & $\mathrm{P}$ & $\mathrm{K}$ & $\mathrm{Ca}$ & $\mathrm{Mg}$ & S & B & $\mathrm{Cu}$ & $\mathrm{Fe}$ & $\mathrm{Mn}$ & $\mathrm{Zn}$ \\
\hline & & \multicolumn{6}{|c|}{$\mathrm{g} \mathrm{kg}^{-1}$} & \multicolumn{5}{|c|}{$\mathrm{mg} \mathrm{kg}^{-1}$} \\
\hline \multirow[t]{4}{*}{ Folha } & LCI & 26,11 & 0,89 & 6,74 & 5,40 & 1,77 & 0,75 & 21,56 & 3,17 & 73,15 & 36,84 & 11,07 \\
\hline & LPI & 28,09 & 1,37 & 7,69 & 5,95 & 1,91 & 1,14 & 25,17 & 5,98 & 104,23 & 84,85 & 16,25 \\
\hline & LPS & 36,08 & 1,97 & 11,52 & 8,18 & 2,47 & 1,63 & 39,37 & 9,56 & 223,40 & 451,47 & 22,84 \\
\hline & LCS & 38,34 & 2,43 & 13,35 & 9,05 & 2,64 & 2,01 & 46,28 & 12,29 & 292,69 & 704,52 & 27,87 \\
\hline \multirow[t]{4}{*}{ Lenho } & LCI & 0,86 & 0,02 & 0,67 & 0,96 & 0,12 & 0,15 & 3,82 & 0,16 & 0,43 & 1,51 & 2,77 \\
\hline & LPI & 1,31 & 0,07 & 1,05 & 1,44 & 0,32 & 0,17 & 4,66 & 0,98 & 3,19 & 2,68 & 4,64 \\
\hline & LPS & 3,11 & 0,27 & 2,48 & 3,40 & 0,57 & 0,27 & 8,01 & 4,18 & 33,42 & 28,13 & 12,10 \\
\hline & LCS & 4,11 & 0,39 & 3,17 & 4,49 & 0,76 & 0,29 & 11,90 & 7,05 & 59,04 & 50,08 & 19,43 \\
\hline \multirow[t]{4}{*}{$\overline{\text { Casca }}$} & LCI & 7,84 & 0,22 & 3,87 & 3,67 & 0,87 & 0,11 & 10,88 & 0,72 & 12,53 & 4,10 & 7,40 \\
\hline & LPI & 9,46 & 0,33 & 5,12 & 6,77 & 1,32 & 0,22 & 14,39 & 1,13 & 25,94 & 10,99 & 11,89 \\
\hline & LPS & 11,51 & 0,47 & 6,70 & 10,72 & 1,89 & 0,35 & 18,85 & 2,75 & 79,35 & 59,92 & 17,60 \\
\hline & LCS & 13,08 & 0,58 & 7,91 & 13,73 & 2,33 & 0,45 & 22,26 & 4,72 & 140,18 & 110,56 & 21,96 \\
\hline \multirow[t]{4}{*}{ Galho morto } & LCI & 5,66 & 0,11 & 0,72 & 3,53 & 1,34 & 0,15 & - & - & - & - & - \\
\hline & LPI & 7,55 & 0,22 & 1,92 & 4,87 & 1,74 & 0,32 & - & -- & - & - & - \\
\hline & LPS & 9,96 & 0,35 & 3,45 & 6,57 & 2,25 & 0,53 & - & - & - & - & -- \\
\hline & LCS & 11,80 & 0,45 & 4,62 & 7,87 & 2,64 & 0,69 & - & - & - & - & - \\
\hline \multirow[t]{4}{*}{$\overline{\text { Galho vivo }}$} & LCI & 3,82 & 0,23 & 2,81 & 2,50 & 0,62 & 0,11 & 9,59 & 0,32 & 6,92 & 3,17 & 7,10 \\
\hline & LPI & 6,90 & 0,57 & 5,16 & 3,73 & 0,80 & 0,31 & 11,69 & 1,26 & 29,49 & 7,24 & 10,93 \\
\hline & LPS & 10,81 & 1,00 & 8,15 & 5,30 & 1,52 & 0,56 & 14,37 & 6,35 & 64,11 & 80,01 & 27,12 \\
\hline & LCS & 13,80 & 1,33 & 10,43 & 6,50 & 2,45 & 0,75 & 16,41 & 12,80 & 112,30 & 165,43 & 49,23 \\
\hline
\end{tabular}

Tabela 6 - Valores de classe obtidos para os limites de controle (LC) e, de precaução (LP) superiores (S) e inferiores (I) para resultados dos teores de nutrientes obtidos em tecidos vegetais de Eucalyptus spp. Santa Maria, - RS, 2007.

Table 6 - Class values obtained for the limits of control (LC), caution (LP) superiors (S) and lower (I) to the levels of nutrients results obtained in vegetable tissues of Eucalyptus spp. Santa Maria Maria,- RS, 2007.

\begin{tabular}{|c|c|c|c|c|c|c|c|c|c|c|c|c|}
\hline \multirow{2}{*}{$\begin{array}{l}\text { Tecido vegetal } \\
\text { Limites }\end{array}$} & & $\mathrm{N}$ & $\mathrm{P}$ & K & $\mathrm{Ca}$ & $\mathrm{Mg}$ & S & B & $\mathrm{Cu}$ & $\mathrm{Fe}$ & Mn & $\mathrm{Zn}$ \\
\hline & & \multicolumn{6}{|c|}{$\mathrm{g} \mathrm{kg}^{-1}$} & \multicolumn{5}{|c|}{$\mathrm{mg} \mathrm{kg}^{-1}$} \\
\hline \multirow[t]{4}{*}{ Folha } & LCI & 22,88 & 1,35 & 9,26 & 7,34 & 2,27 & 1,23 & 30,42 & 6,45 & 62,58 & 768,57 & 17,63 \\
\hline & LPI & 23,31 & 1,38 & 9,54 & 7,56 & 2,33 & 1,25 & 31,57 & 6,68 & 65,53 & 818,38 & 18,22 \\
\hline & LPS & 25,03 & 1,52 & 10,64 & 8,45 & 2,54 & 1,33 & 36,21 & 7,59 & 77,48 & 1020,17 & 20,61 \\
\hline & LCS & 25,47 & 1,55 & 10,92 & 8,67 & 2,59 & 1,35 & 37,39 & 7,82 & 80,51 & 1071,26 & 21,21 \\
\hline \multirow[t]{4}{*}{ Lenho } & LCI & 1,70 & 0,15 & 2,09 & 1,06 & 0,41 & 0,19 & 3,49 & 2,12 & 30,60 & 49,88 & 3,06 \\
\hline & LPI & 1,83 & 0,18 & 2,26 & 1,18 & 0,43 & 0,20 & 3,90 & 2,35 & 34,06 & 64,31 & 3,79 \\
\hline & LPS & 2,35 & 0,32 & 2,94 & 1,68 & 0,55 & 0,26 & 5,52 & 3,25 & 47,92 & 122,03 & 6,71 \\
\hline & LCS & 2,48 & 0,36 & 3,11 & 1,80 & 0,57 & 0,27 & 5,93 & 3,48 & 51,39 & 136,46 & 7,44 \\
\hline \multirow[t]{4}{*}{ Casca } & LCI & 4,20 & 0,48 & 4,98 & 18,98 & 2,80 & 0,32 & 18,09 & 2,87 & 33,36 & 801,20 & 7,03 \\
\hline & LPI & 4,32 & 0,49 & 5,24 & 20,05 & 2,97 & 0,33 & 18,96 & 3,04 & 36,76 & 869,66 & 7,74 \\
\hline & LPS & 4,82 & 0,57 & 6,30 & 24,37 & 3,63 & 0,41 & 22,44 & 3,76 & 50,38 & 1143,52 & 10,62 \\
\hline & LCS & 4,94 & 0,58 & 6,56 & 25,44 & 3,80 & 0,42 & 23,31 & 3,93 & 53,78 & 1211,98 & 11,33 \\
\hline \multirow[t]{4}{*}{ Galho vivo } & LCI & 3,81 & 0,44 & 4,29 & 8,81 & 1,47 & 0,30 & 10,94 & 4,97 & 28,44 & 442,07 & 9,25 \\
\hline & LPI & 3,99 & 0,48 & 4,51 & 9,48 & 1,54 & 0,31 & 11,34 & 5,23 & 31,44 & 486,23 & 10,07 \\
\hline & LPS & 4,75 & 0,66 & 5,43 & 12,12 & 1,82 & 0,39 & 12,92 & 6,29 & 43,42 & 662,87 & 13,35 \\
\hline & LCS & 4,93 & 0,70 & 5,65 & 12,79 & 1,89 & 0,40 & 13,32 & 6,55 & 46,42 & 707,03 & 14,17 \\
\hline
\end{tabular}

Revista Árvore, Viçosa-MG, v.34, n.3, p.529-537, 2010 
Tabela 7 - Valores de classe obtidos para os limites de controle (LC), ) e de precaução (LP) superiores (S) e inferiores (I) para resultados dos teores de nutrientes obtidos em tecidos vegetais de Pinus taeda. Santa Maria, - RS, 2007.

Table 7 - Class values obtained for the limits of control (LC), caution (LP) superiors (S) and lower (I) to the levels of nutrients results obtained in vegetable tissues of Pinus taeda. Santa Maria, - RS, 2007.

\begin{tabular}{|c|c|c|c|c|c|c|c|c|c|c|c|c|}
\hline \multirow[t]{2}{*}{ Tecido vegetal } & \multirow[b]{2}{*}{ Limites } & $\mathrm{N}$ & $\mathrm{P}$ & $\bar{K}$ & $\mathrm{Ca}$ & $\mathrm{Mg}$ & $S$ & B & $\mathrm{Cu}$ & $\mathrm{Fe}$ & Mn & $\mathrm{Zn}$ \\
\hline & & \multicolumn{6}{|c|}{$\mathrm{g} \mathrm{kg}^{-1}$} & \multicolumn{5}{|c|}{$\mathrm{mg} \mathrm{kg}^{-1}$} \\
\hline \multirow[t]{4}{*}{ Acícula } & LCI & 7,09 & 0,65 & 1,51 & 1,68 & 0,82 & 0,34 & 6,10 & 2,67 & 56,45 & 0,72 & 15,39 \\
\hline & LPI & 8,52 & 0,90 & 2,58 & 2,57 & 0,96 & 0,53 & 13,60 & 3,78 & 124,80 & 165,54 & 20,25 \\
\hline & LPS & 14,26 & 1,20 & 6,82 & 3,69 & 1,50 & 1,31 & 22,94 & 5,16 & 209,74 & 370,36 & 26,27 \\
\hline & LCS & 15,69 & 1,45 & 7,89 & 4,58 & 1,64 & 1,50 & 30,44 & 6,27 & 278,09 & 535,18 & 31,13 \\
\hline \multirow[t]{4}{*}{ Lenho } & LCI & 1,11 & 0,07 & 0,27 & 0,59 & 0,22 & 0,31 & 3,91 & 1,32 & 11,17 & 30,92 & 3,61 \\
\hline & LPI & 1,21 & 0,08 & 0,47 & 0,63 & 0,24 & 0,33 & 4,45 & 1,53 & 15,87 & 40,40 & 4,48 \\
\hline & LPS & 1,65 & 0,14 & 0,73 & 0,77 & 0,32 & 0,43 & 6,61 & 2,39 & 34,69 & 78,34 & 7,98 \\
\hline & LCS & 1,75 & 0,15 & 0,93 & 0,81 & 0,34 & 0,45 & 7,15 & 2,60 & 39,39 & 87,82 & 8,85 \\
\hline \multirow[t]{4}{*}{ Casca } & LCI & 2,48 & 0,21 & 0,73 & 0,78 & 0,36 & 0,34 & 8,90 & 2,66 & 23,30 & 30,74 & 12,22 \\
\hline & LPI & 2,80 & 0,25 & 0,96 & 1,01 & 0,44 & 0,37 & 9,99 & 3,75 & 113,86 & 35,83 & 15,50 \\
\hline & LPS & 4,10 & 0,45 & 1,86 & 1,95 & 0,74 & 0,51 & 14,35 & 5,11 & 226,40 & 56,17 & 28,60 \\
\hline & LCS & 4,42 & 0,49 & 2,09 & 2,18 & 0,82 & 0,54 & 15,44 & 6,20 & 316,96 & 61,26 & 31,88 \\
\hline \multirow[t]{4}{*}{ Galho vivo } & LCI & 0,93 & 0,08 & $-0,10$ & 0,64 & 0,31 & 0,23 & 5,70 & 1,54 & 15,54 & 10,56 & 7,47 \\
\hline & LPI & 2,38 & 0,18 & 0,41 & 1,01 & 0,48 & 0,30 & 8,10 & 3,71 & 52,10 & 88,24 & 13,57 \\
\hline & LPS & 4,18 & 0,58 & 2,43 & 2,51 & 0,68 & 0,60 & 11,08 & 6,41 & 97,52 & 184,78 & 21,15 \\
\hline & LCS & 5,63 & 0,68 & 2,94 & 2,88 & 0,85 & 0,67 & 13,48 & 8,58 & 134,08 & 262,46 & 27,25 \\
\hline \multirow[t]{4}{*}{ Galho morto } & LCI & 0,69 & 0,01 & 0,04 & 0,85 & 0,23 & 0,12 & 6,31 & 1,11 & 19,87 & 13,35 & 1,71 \\
\hline & LPI & 1,46 & 0,05 & 0,16 & 1,34 & 0,34 & 0,26 & 8,41 & 3,11 & 82,04 & 44,49 & 3,79 \\
\hline & LPS & 2,42 & 0,21 & 0,32 & 3,32 & 0,46 & 0,44 & 11,03 & 5,59 & 159,30 & 284,03 & 19,77 \\
\hline & LCS & 3,19 & 0,25 & 0,44 & 3,82 & 0,57 & 0,58 & 13,13 & 7,59 & 221,47 & 343,92 & 23,77 \\
\hline
\end{tabular}

do limite de precaução e, principalmente, do limite de controle, são provenientes de amostras contaminadas ou de resultados de erros de determinação, medição ou análise, devido a possíveis calibragens fora das especificações dos equipamentos ou descuidos do responsável pela análise, indicando, assim, eventuais descontroles no processo de análise. Feigenbaum (1994) relatou que, com os limites de precaução e de controle estabelecidos, algumas aplicações são recomendadas para determinar o grau de controle do processo, realizar a predição de rejeições antes da produção de resultados “não conformes”, analisar o desempenho da atividade, estabelecer as tolerâncias e os custos de re análise e definir diretivas para gerenciamento do processo de controle de qualidade.

Assim, concluiu-se que os teores dos elementos químicos são diferentes entre Acacia mearnsii, Eucalyptus spp. e Pinus taeda para cada tipo de componente vegetal analisado. Nas folhas e acículas houve a maior concentração dos elementos químicos, enquanto no lenho, as menores. Os limites estimados, propostos no trabalho, permitem maior controle na classificação dos resultados, aumentando a confiabilidade nas análises nutricionais.

\section{AGRADECIMENTOS}

Ao CNPq, pelos recursos financeiros e pelas bolsas de pós-graduação e de produtividade em pesquisa.

\section{REFERÊNCIAS}

ANDRAE, F.; KRAPFENBAUER, A. Estudos da situação da biomassa e nutrientes de um reflorestamento de quatro anos com Eucalyptus saligna Smith em Santa Maria-RS. In:ANDRAE, F.; KRAPFENBAUER, A. Pesquisa austríaco-brasileira (1973-1982). Santa Maria: 1983. p.68-85.

ARACRUZ CELULOSE S.A. Sistema de gestão da qualidade: macroprocesso: sistema de gestão, microprocesso: gestão da qualidade. sistema de documentos. Guaíba: ARCEL, 2004.

BARRICHELO, L. R. Quantificação da biomassa e dos nutrientes em floresta de Acacia mearnssi De Wild. na Região Sul. 2003. 88f. Dissertação (Mestrado em Engenharia Florestal) - Universidade Federal de Santa Maria, Santa Maria, 2003. 
BELLOTE, A. F. J.; SILVA, H. D. Sampling techniques and nutritional evaluations in eucalypt plantations. In: GONÇALVES, J. L. M.; BENEDETTI, V. (Eds.) Forest nutrition and fertilization. Piracicaba: IPEF, 2004. p.113-139.

BINKLEY, D. Forest nutrition management. New York: A Wiley-Interscience/ John Wiley, 1986. 290p.

BIZON, J. M. C. Avaliação da sustentabilidade nutricional de plantios de Pinus taeda L. usando um balanço de entrada-saída de nutrientes. 2005. 95f. Dissertação (Mestrado em Recursos Florestais) - Escola Superior de Agricultura Luiz de Queiroz, Piracicaba, 2005.

BRAVO, P. C. Controle estatístico da qualidade. In: REUNIÃO ANUAL DA REGIÃO BRASILEIRA DA SOCIEDADE DE BIOMETRIA E SIMPÓSIO DE ESTATÍSTICAAPLICADA À EXPERIMENTAÇÃO AGRONÔMICA, 40., 1995. Ribeirão Preto. Anais... Ribeirão Preto: Universidade de São Paulo, 1995. 71p.

BURGER, L. M.; RICHTER, H. G. Anatomia da madeira. São Paulo: Nobel, 1991. 154p.

CALDEIRA, M. V. W. et al. Ciclagem de nutrientes em Acacia mearnsii DE WILD. V. Quantificação do conteúdo de nutrientes na biomassa aérea de Acacia mearnsii DE WILD. procedência australiana. Ciência Rural, v.30, n.6, p.977-982, 2000.

CAMPOS, H. Estatística experimental nãoparamétrica. 4.ed. Piracicaba: Escola Superior de Agricultura Luiz de Queiroz, 1983. 349p.

CANTARELLA, H.; QUAGGIO, J. A.; ANDRADE, J. C. Controle de qualidade dos resultados analíticos. In: GONÇALVES, J. L. M.; BENEDETTI, V. (Eds.) Análise química de solos tropicais. 2.ed. São Paulo, Instituto Agronômico, 2001. p.123-132.

DE HOOG, R. J. Site-nutrition-growth relationships of Araucaria angustifolia (Bert.) O. Ktze. in southern Brazil. 1981. 161f. (Inaugural-Dissertation) Universidade zu Freiburg, Freiburg, 1981.
DELL, B.; MALAJACZUK, N.; GROVE, T. S. Nutrient disorders in plantation eucalypts. Canberra: Australian Centre for International Agricultural Research, 1995. 104p.

ELEOTERIO, J. R.; STORCK, L.; LOPES, S. J. Caracterização de peças de madeira produzidas em serraria visando o controle de qualidade.

Ciência Florestal, v.6, n.1, p.89-99, 1996.

FEIGENBAUM, A. V. Controle da qualidade total: métodos estatísticos aplicados à qualidade. São Paulo: Makron Books, 1994. 379p.

GONÇALVES, J. L. M. Recomendação de adubação para Eucalyptus, Pinus e espécies típicas da Mata Atlântica. Documentos Florestais, n.15, p.1-23, 1995.

GONÇALVES, J. L. M. et al. An evaluation of minimum and intensive soil preparation regarding fertility and tree nutrition. In: GONÇALVES, J. L. M. \& BENEDETTI, V. (Eds.) Forest nutrition and fertilization. Piracicaba: IPEF, 2004. p.13-64.

JACOVINE, L. A. G. et al. Avaliação da qualidade operacional em cinco subsistemas de colheita florestal. Revista Árvore, v.29, n.3, p.391-400, 2005.

LORENTZ, L. H.; FORTES, F. O.; LÚCIO, A. D. Análise de trilha entre as variáveis das análises de sementes de espécies florestais exóticas do Rio Grande do Sul. Revista Árvore, v.30, n.4, p. 567-574, 2006.

LÚCIO, A. D.; STORCK, L.; BANZATTO, D. A. Classificação dos experimentos de competição de cultivares quanto à sua precisão. Pesquisa Agropecuária Gaúcha, v.5, n.1, p.99-103, 1999.

MALAVOLTA, E.; VITTI, G. C.; OLIVEIRA, S. A. Avaliação do estado nutricional de plantas: princípios e aplicações. 2.ed. Piracicaba: Potafos, 1997. 319p.

MARSCHNER, H. Mineral nutrition of higher plants. 2.ed. London, Academic Press, 1995. 889p.

NEVES, J. C. L. Produção e partição de biomassa, aspectos nutricionais e hídricos em plantios clonais de eucalipto na região litorânea do Espírito Santo. 2000. 191f. Tese (Doutorado em Produção Vegetal) - Universidade Estadual do Norte Fluminense, Campos dos Goytacazes, 2000. 
PALLARDY, S. Physiology of woody plants. San Diego: Academic Press, 2008. 454p.

REIS, M. G. F.; BARROS, N. F. Ciclagem de nutrientes em plantios de eucalipto. In: BARROS, N. F.; NOVAIS, R. F. (Eds). Relação solo eucalipto. Viçosa, MG: Folha de Viçosa, 1990. p.265-302.

REISSMANN, C. B. et al. Metodologia da amostragem e análise das acículas de Araucaria angustifolia: influência da idade das acículas. Floresta, v.1, n.7, p.5-12, 1976.

SAEG. Sistema para análises estatísticas, Versão 9.1. Viçosa, MG: Fundação Arthur Bernardes; Universidade Federal de Viçosa, 2007.

SCHUMACHER, M. V. et al. Biomassa e

distribuição de nutrientes em um povoamento de Pinus taeda em Cambará do Sul, RS. In: SIMPOSIO BRASILEIRO DE PÓS-GRADUAÇÃO EM ENGENHARIA FLORESTAL, 2., 2002, Viçosa, MG. Anais... Viçosa, MG, 2002. 652p.
SILVA, H. D. Modelos matemáticos para a estimativa da biomassa e do conteúdo de nutrientes em plantações de Eucalyptus grandis de diferentes idades. 1996. 101f. Tese (Doutorado em Engenharia Florestal) - Universidade Federal do Paraná, Curitiba, 1996.

SIMÕES, J. W.; COUTO, H. T. Z.; KAJIYA, S. Tolerância da Araucaria angustifolia a teores crescentes de alumínio. Piracicaba: IPEF, n.6, p.93-102, 1973.

WITSCHORECK, R. Biomassa e nutrientes no corte raso de um povoamento de Pinus taeda $\mathrm{L}$. de 17 anos de idade no município de Cambará do Sul - RS. 2008. 80f.

Dissertação (Mestrado e Engenharia Florestal) -

Universidade Federal de Santa Maria, Santa Maria, 2008.

ZEN, S.; POGGIANI, F.; COUTO, H. T. Z. Variação na concentração de nutrientes ao longo do caule de Eucalyptus saligna: implicações na utilização energética dos resíduos florestais. Piracicaba: IPEF, 1981. 7p. (Circular Técnica, 136). 